\title{
Tenfold improved sensitivity using high refractive-index substrates for surface plasmon sensing
}

\section{$\operatorname{AUTHOR}(\mathrm{S})$ :}

Micheletto, Ruggero; Hamamoto, Katsumi; Fujii, Takashi; Kawakami, Yoichi

\section{CITATION:}

Micheletto, Ruggero ...[et al]. Tenfold improved sensitivity using high refractive-index substrates for surface plasmon sensing. APPLIED PHYSICS LETTERS 2008, 93(17): 174104.

\section{ISSUE DATE:}

2008-10-27

URL:

http://hdl.handle.net/2433/84580

\section{RIGHT:}

Copyright 2008 American Institute of Physics. This article may be downloaded for personal use only. Any other use requires prior permission of the author and the American Institute of Physics. 


\title{
Tenfold improved sensitivity using high refractive-index substrates for surface plasmon sensing
}

\author{
Ruggero Micheletto, ${ }^{1,2, a)}$ Katsumi Hamamoto, ${ }^{2,3}$ Takashi Fujii, ${ }^{4}$ and Yoichi Kawakami ${ }^{2}$ \\ ${ }^{1}$ Nanoscience and Technology, International Graduate School of Art and Sciences, Yokohama City University, \\ Seto, Kanazawa-ku, Yokohama 236-0027, Japan \\ ${ }^{2}$ Department of Electronic Science, Graduate School of Engineering, Kyoto University, Nishigyo-ku, \\ Katsura, 615-8510 Kyoto, Japan \\ ${ }^{3}$ Teramecs Ltd., 97 Higashi Koyanouchi-cho, Takeda, Fushimi-ku, 612-8448 Kyoto, Japan \\ ${ }^{4}$ Murata Manufactoring Co., Ltd., Higashikotari 1-chome, Nagaokakyo-shi, 617-8555 Kyoto, Japan
}

(Received 25 June 2008; accepted 1 October 2008; published online 31 October 2008)

\begin{abstract}
Surface plasmon resonance sensors exploit the high sensitivity to local perturbations of plasma waves in a thin metal layer. These devices have a wide range of applications as biomedical, environmental, industrial, and homeland security. We concentrate on the theoretical aspects of the sensing principle. By calculations at various indexes of refraction we proved that using substrate material of higher index, sensitivity and dynamics range improve conspicuously. Finally, we show experimental data taken using a special transparent ceramic material of exceptionally high index of refraction $n=2.04$. Tests demonstrate sensitivity about one order of magnitude better than those obtained with conventional BK7 glass. (C) 2008 American Institute of Physics.
\end{abstract}

[DOI: $10.1063 / 1.3005584$ ]

Surface plasmon resonance (SPR) sensors have been developed to study chemical reactions occurring in the proximity of a metal surface. ${ }^{1-3}$ A SPR setup can be arranged in various configurations. ${ }^{4-9}$ Most commonly, the sample substrate consists of a thin gold layer deposited on a glass prism where light beam is shone in total internal reflection condition (Kretschmann configuration ${ }^{1}$ ). The light reflected is detected by a charge coupled device (CCD) camera that integrates the light spot [Fig. 1(a)]. At a certain angle of incidence, reflectivity drops sharply nearly to zero because of the SPR effect. This phenomenon is sensible to any perturbation of conditions nearby the metal layer, most importantly refractive index (RI) changes. Generally, the device is enclosed in a flow cell. The metal layer is functionalized by a ligand able to bind to target sample molecules. Overall RI changes depend on sample index of refraction, sample thickness, and coverage. Thus, molecular complex formed on the layer will be perturbing the resonance. Very high sensitivity to the thickness and coverage allows real time study of the formation of molecular complexes. This methodology is usually called SPR sensing and it is applied mostly in biomedical, environmental, and industrial applications. ${ }^{10}$

In our treatment the calculation of the surface plasmon effect is reduced to a well-known two-dimensional three media Fresnel theory. We define $\epsilon_{p}, \epsilon_{m}$, and $\epsilon_{s}$ as the dielectric constants of the three media $p, m$, and $s$. The prism is symbolized by the index $p$, the metal by $m$, and the sample (material deposited over the metal) by $s$. We have

$$
\epsilon_{m}=\epsilon_{m}^{\infty}-\frac{\omega_{p}^{2}}{\omega\left(\omega+i \omega_{\tau}\right)},
$$

with $\epsilon_{m}^{\infty}=9.75, \quad \omega_{p}=1.36 \times 10^{16} \mathrm{rad} / \mathrm{s}, \quad$ and $\quad \omega_{\tau}=1.45$ $\times 10^{14} \mathrm{rad} / \mathrm{s}$, in the case of gold. ${ }^{11}$

The total reflectivity in the three layers system is given by the relation

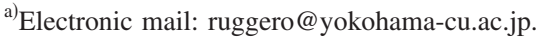

$$
R=\left|\frac{r_{p m}+r_{m s} e^{2 i k_{m z} d}}{1+r_{p m} r_{m s} e^{2 i k_{m z} d}}\right|^{2},
$$

where

$$
\begin{gathered}
r_{p m}=\frac{k_{p z} \epsilon_{m}-k_{m z} \epsilon_{p}}{k_{p z} \epsilon_{m}+k_{m z} \epsilon_{p}}, \\
r_{m s}=\frac{k_{m z} \epsilon_{s}-k_{s z} \epsilon_{m}}{k_{m z} \epsilon_{s}+k_{s z} \epsilon_{m}} .
\end{gathered}
$$

The other parameters appearing in the relation are $k_{j z}$ $=\sqrt{\epsilon_{j}\left(\omega^{2} / c^{2}\right)-k_{x}^{2}} \mid j=p, m, s$ and $k_{x}=\sqrt{\epsilon_{p}}(\omega / c) \sin \alpha$. The index $j$ $=p, m$, and $s$ indicates that the symbol $j$ stands for $p, m$, and $s$; a compact representation of the three parameters $k_{p z}, k_{m z}$, and $k_{s z}$.
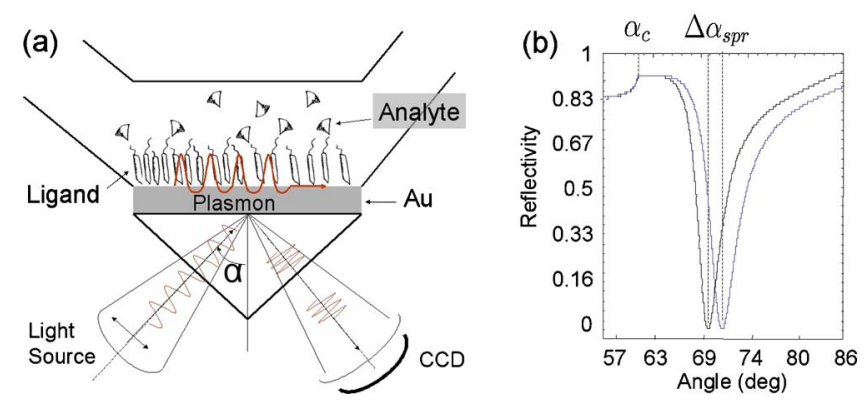

FIG. 1. (Color online) (a) A schematic view of the core of a generic SPR system. A transparent prism is metal coated on one face where a light beam is shone in total internal reflection conditions. Plasmon Resonance is detected monitoring with a CCD camera the reflectivity properties of the metal surface. This surface is treated with a ligand. When a liquid containing the target molecule is circulated, analyte, and ligand bind to form a larger complex. As a result the overall RI and the reflectivity curve are changed. (b) Reflectivity SPR curve as integrated by the CCD camera in the scheme (a). The curve is calculated for a gold coating of about $52 \mathrm{~nm}$, in water solution and illuminated by $670 \mathrm{~nm}$ laser beam. A positive variation in index of refraction (about $1 \%$ ) results in an increased resonance angle. 


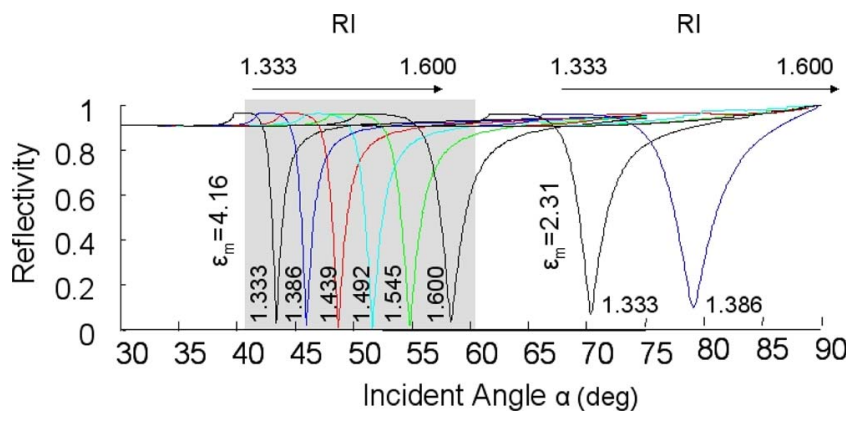

FIG. 2. (Color online) The comparative analysis of the response of a glass prism based SPR sensor and a higher refraction index material based one. The grayed group of curves is the simulated SPR response to different RI samples, ranging from $n=1.333$ to $n=1.6$. The calculation are made considering a $\lambda=670 \mathrm{~nm}$ laser beam, a $d=50 \mathrm{~nm}$ thin gold metal layer over a $n$ $=2.04\left(\epsilon_{m}=4.16\right)$ prism. The other curves are calculated in the same conditions for a standard $n=1.52\left(\epsilon_{m}=2.31\right)$ glass prism. In the case of glass substrate only the first two lower RI curves show resonance peaks. With high RI material deeper and sharper peaks appear for all the RI range. In an actual sensor this will result in wider dynamic, better signal-to-noise ratio and sensitivity.

The reflectivity profile obtained is shown in Fig. 1(b) for two slightly different values of $\boldsymbol{\epsilon}_{s}$.

In Fig. 2 are shown the response curves for different sample RIs, calculated for a glass prism $\left(\epsilon_{m}=2.31\right)$ and for a high RI material $\left(\epsilon_{m}=4.16\right)$. The clear advantages in the latter case are evident. For glassy prism (right group of curves), the resonance profile is less sharp and less deep. This results in decreased sensitivity and signal to noise ratio. The simulation is performed in both cases for samples of RI ranging between $n=1.333$ and $n=1.6$, however, only the first two resonance peaks are observable. Instead, the prism of higher RI material gives very deep and sharp resonance peaks for all the RI range.

To confirm these theoretical insights we performed a test with a substrate of high RI. We used a transparent ceramic material of $n=2.04$ RI (Lumicera ${ }^{\mathrm{TM}}$ ceramics, Murata Manufacturing Co., Ltd.). This material is of low cost and can be molded to any shape; its transmittance spectrum is shown in Fig. 3.

Normally, ceramics are opaque because of the existence of pores and impurity phases at the grain boundary, however in this material the entire production process has been optimized in order to realize a dense and homogeneous

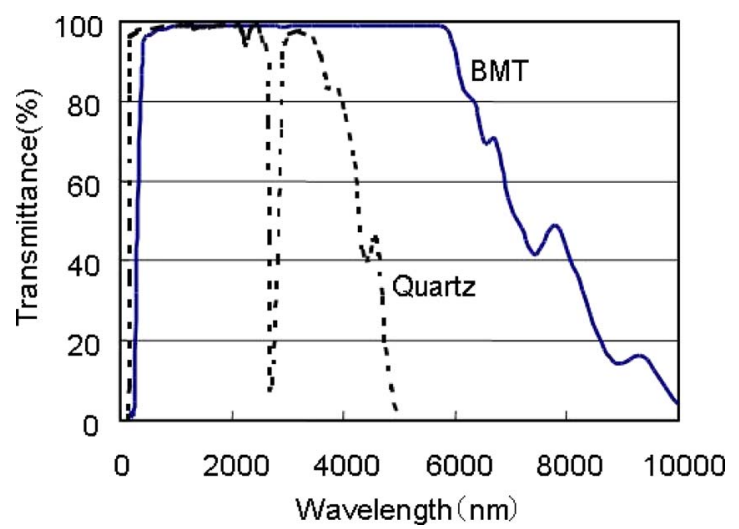

FIG. 3. (Color online) The transmission spectra of the ceramic used is compared with quartz. This is a special transparent material based on the so called BMT mixture: $\mathrm{Ba}(\mathrm{Sn}, \mathrm{Zr}, \mathrm{Mg}, \mathrm{Ta}) \mathrm{O} 3$. This ceramic can be molded to any shape, has a very high RI $(n=2.04)$ and a wide transmission range.
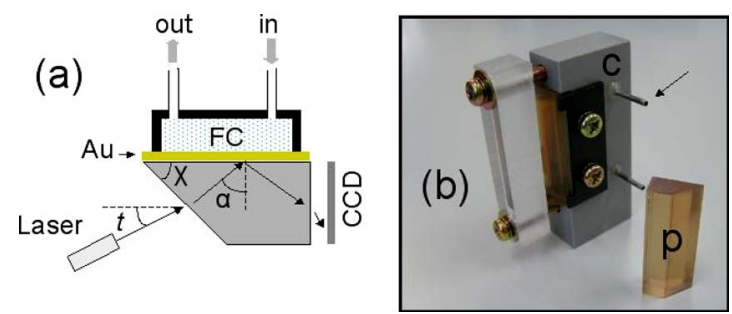

FIG. 4. (Color online) The core of the setup. (a) A schematic representation of the ceramic sensor in the flow cell. A $\lambda=670 \mathrm{~nm}$ diode laser illuminates a $52 \mathrm{~nm}$ thick gold layer deposited on the face of the sensor exposed to the solution circulating in the flow cell. The laser beam is incident at a variable angle $t$, and it is internally reflected on the gold surface at $\alpha$. The reflected light is finally collected by a detector (CCD). The angle $\chi$ is $45^{\circ}$. (b) A picture of the sensor. The letter "c" indicates the flow-cell block, one of the in/out pipes is visible to the right indicated by an arrow. The ceramic "prism" sensor is encased in the center, recognizable by its reddish color. To the side of the flow cell another isolated one is shown marked by the symbol "p."

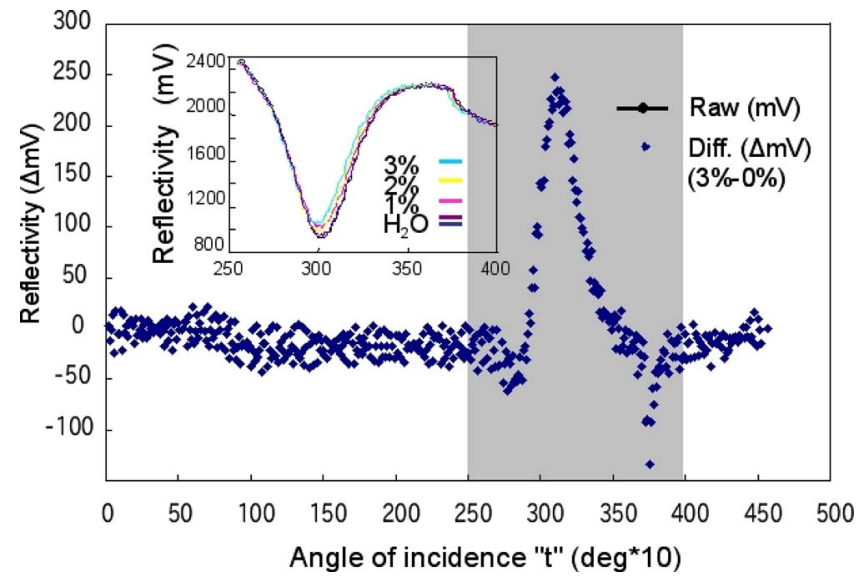

FIG. 5. (Color online) The differential response of the BMT ceramic based sensor. The signal plotted results from the reflectivity difference between pure water and a $3 \% w / w \%$ sucrose solution. In the inset the raw reflectivity curves are shown within the angular range grayed on the main plot.

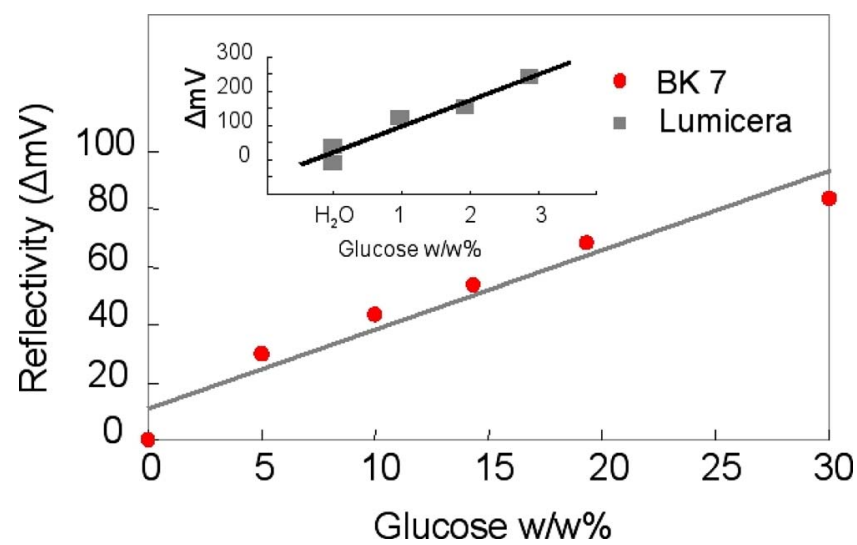

FIG. 6. (Color online) The SPR sensor linear response to different glucose concentration of a standard glassy SPR sensor. In the inset, differential response for ceramic substrate reaches about $250 \mathrm{mV}$ at $3 \%$ glucose concentration, one order of magnitude better than with the glass based sensor. 
ceramic. ${ }^{12,13}$ For reason of compatibility with our flow cells, the shape of the sensor is not a perfect prism as in classic Kretschmann configuration. In Fig. 4 a sketch of the sensor and the beam optical path are shown.

Using four samples at low glucose concentration, we scan the angle if incidence $t$ (defined in Fig. 4) from $0^{\circ}$ to $90^{\circ}$, obtaining the series of curves shown in the inset of Fig. 5 , note that the angle $t=\chi / 4-\sin ^{-1}\left[n_{1} / n_{0} \sin (\alpha-\chi)\right]$ runs in opposite direction to $\alpha$.

This results are compared to the SPR response of a normal BK7 glassy SPR sensor in Fig. 6. Both systems show good linearity and high sensitivity. However, the ceramic based SPR show about $250 \mathrm{mV}$ differential response between water and $3 \%$ glucose solution. This value is one order of magnitude higher than the BK7 glass case.

By a basic theoretical investigation we showed that high index of refraction materials can be used in SPR sensors to obtain higher sensitivity and wider dynamic ranges. We simulated performances of ideal SPR tests showing comparative plots proving better performances. Also, we could setup a real test using a transparent ceramic of refraction index $n$ $=2.04$, shaped as a prism and sputtered with gold for SPR sensing. We obtained good linearity and higher sensitivity, about one order of magnitude better than with conventional glass-based SPR sensors.

${ }^{1}$ E. Kretschmann, Z. Phys. 241, 313 (1971).

${ }^{2}$ R. C. Jorgenson and S. S. Yee, Sens. Actuators B 12, 213 (1993).

${ }^{3}$ P. B. Daniels, J. K. Deacon, M. J. Eddowes, and D. G. Pedley, Sens. Actuators 15, 11 (1988).

${ }^{4}$ K. Hamamoto, R. Micheletto, M. Oyama, A. A. Umar, S. Kawai, and Y. Kawakami, J. Opt. A, Pure Appl. Opt. 8, 268 (2006).

${ }^{5}$ K. Kurihara, K. Nakamura, E. Hirayama, and K. Suzuki, Anal. Chem. 74, 6323 (2002).

${ }^{6}$ M. Malmqvist, Nature (London) 361, 186 (1993).

${ }^{7}$ K. Nakatani, S. Sando, and I. Saito, Nat. Biotechnol. 19, 51 (2001).

${ }^{8}$ J.-F. Masson, T. M. Battaglia, M. J. Davidson, Y. C. Kim, A. M. C. Prakash, and S. B. K. S. Beaudoin, Talanta 67, 908 (2005).

${ }^{9}$ Y. Li, J. Sun, L. Wang, P. Zahn, Z. Cao, and Z. Wang, Appl. Phys. A: Mater. Sci. Process. 92, 291 (2008).

${ }^{10}$ K. Tamada and W. Knoll, Mirai Zairyo 8, 22 (2008).

${ }^{11}$ K. Kurihara, K. Nakamura, and K. Suzuki, Sens. Actuators B 86, 49 (2002)

${ }^{12}$ N. Tanaka, Y. Kintaka, S. Kuretake, and N. Wada, Material Integration 19, 7 (2006).

${ }^{13}$ M. Kuwabara, Materia 39, 132 (2000). 